\title{
Catalytic Alkylation of 2-Methylfuran with Formalin Using Supported Acidic Ionic Liquids
}

\author{
Li, Hu; Shunmugavel, Saravanamurugan; Yang, Song; Riisager, Anders
}

Published in:

A C S Sustainable Chemistry \& Engineering

Link to article, DOI:

$10.1021 /$ acssuschemeng.5b00850

Publication date:

2015

Document Version

Peer reviewed version

Link back to DTU Orbit

Citation (APA):

Li, H., Shunmugavel, S., Yang, S., \& Riisager, A. (2015). Catalytic Alkylation of 2-Methylfuran with Formalin Using Supported Acidic lonic Liquids. A C S Sustainable Chemistry \& Engineering, 3(12), 374-3280. https://doi.org/10.1021/acssuschemeng.5b00850

\section{General rights}

Copyright and moral rights for the publications made accessible in the public portal are retained by the authors and/or other copyright owners and it is a condition of accessing publications that users recognise and abide by the legal requirements associated with these rights.

- Users may download and print one copy of any publication from the public portal for the purpose of private study or research.

- You may not further distribute the material or use it for any profit-making activity or commercial gain

- You may freely distribute the URL identifying the publication in the public portal 


\title{
Catalytic alkylation of 2-methylfuran with formalin using supported acidic ionic liquids
}

\author{
$\mathrm{Hu} \mathrm{Li}^{a, b}$, Shunmugavel Saravanamurugan ${ }^{a}$, Song Yang $^{b}$, and Anders Riisager ${ }^{a} *$ \\ ${ }^{a}$ Centre for Catalysis and Sustainable Chemistry, Department of Chemistry, Technical University of \\ Denmark, DK-2800 Kgs. Lyngby, Denmark \\ ${ }^{b}$ State-Local Joint Engineering Laboratory for Comprehensive Utilization of Biomass, Center for R\&D \\ of Fine Chemicals, Guizhou University, Guiyang 550025, PR China
}

* Corresponding Author: A. Riisager, Email: ar@kemi.dtu.dk; Tel.: +45 45252233; Fax: +45 45883136.

\begin{abstract}
Biphasic alkylation of 2-methylfuran (2-MF) with formalin was carried out with a series of SBA-15 supported acidic ionic liquid catalysts (acidic SILCs) under mild reaction conditions. Acidic SILC with sulfonic acid groups $\left(\mathrm{SO}_{3} \mathrm{H}\right)$ and long alkyl chains was observed to have higher catalytic activity than commercial sulfonic acid resin catalysts for the alkylation reaction in terms of TONs/TOFs as well as selectivity $(90 \%)$ towards the $\mathrm{C}_{11}$ oxygenate bis(5-methylfuran-2-yl)methane (BMFM). The reaction product was easily separated by addition of the non-polar solvent $n$-heptane and additional water to form a biphasic system. The reactivity of other biomass-based substrates such as 3,4-dimethoxybenzaldehyde, furfural, glycolaldehyde and glyceraldehyde were also investigated over acidic SILCs, and excellent yields of about $80 \%$ or higher were obtained of the corresponding condensed products (except from glyceraldehyde). Readily catalyst recovery from the aqueous phase after extraction of BMFM with $n$-heptane and reusability for at least four consecutive reaction runs without significant loss of catalyst activity was further exemplified for a selected catalyst.
\end{abstract}

Keywords: Alkylation; 2-Methylfuran; Formalin; Supported acidic ionic liquids catalysts (SILCs); SBA-15; Solid acids 


\section{Introduction}

Conversion of terrestrial biomass-derived substrates into fuels and useful chemicals has progressively been studied in the last decade in order to find potential alternatives and substitutes for current fossil-based fuels and chemicals. ${ }^{1-3}$ The direct conversion of cellulose/sugars into fuels or targeted chemicals such as, for example gamma-valerolactone, in high yield is however generally challenging due to the presence of reactive multi-functional groups, non-selective reaction pathways, different optimal reaction conditions for each step etc. ${ }^{4,5}$ In this connection, the transformation of sugar-derived intermediates, such as furfural and methyl furan, into selective products with increased number of carbon atoms (hydrocarbon fuel precursors) are thus very important. ${ }^{6}$ Fatty acid methyl esters (FAMEs) - biodiesel have been promoted widely as a substitute for fossil-based fuels. However, since biodiesel production most often rely on vegetable oils which are also food resources, biodiesel is gradually getting demoted as fuel. ${ }^{6}$ In this context, the conversion of sugar-derived intermediates to oxygenated products - which can further be converted into liquid alkane fuels by deoxygenation - have received increased attention. ${ }^{7-9}$

Liquid $\mathrm{C}_{7}-\mathrm{C}_{15}$ alkanes can be produced by dehydration of biomass-derived carbohydrates followed by aldol condensation/dehydration/hydrogenation over multi-catalytic systems in aqueous phase..$^{5}$ Acetone-butanol-ethanol (ABE) fermentation is a well-known and important route to produce $n$-butanol as a major product from glucose. ${ }^{10} n$-Butanol can then be dehydrated to produce 1-butene, ${ }^{11}$ and a fully saturated fuel mixture can be obtained through successive oligomerization and hydrogenation over solid acid catalysts and $\mathrm{PtO}_{2}$, respectively, in the absence of solvents. ${ }^{12,13}$ Another interesting route to make long chain alkane fuels are via aldol condensation of furfural and acetone catalyzed by mineral base, ${ }^{8}$ organic base ${ }^{14}$ or solid base catalysts. ${ }^{15,16}$ Here $\mathrm{C}_{8}$ and $\mathrm{C}_{13}$ oxygenates were obtained in high yields, and metal loaded catalysts could efficiently promote the subsequent hydrogenation and hydrodeoxygenation (HDO) to afford $\mathrm{C}_{8}-\mathrm{C}_{13}$ alkanes. ${ }^{17-19} \mathrm{~A}$ different reaction approach reported to increase the number of carbon atoms in fuel products involve 
benzoin coupling or self-coupling of furanic aldehydes using $N$-heterocyclic carbene catalysts or reductants. ${ }^{20-22}$ In these processes, moderate to high yields of straight or branched $\mathrm{C}_{8}-\mathrm{C}_{14}$ alkanes were obtained after $\mathrm{HDO}$ reaction.

Hydrogenolysis of furfural - a dehydration product of xylose - can lead to formation of 2-methylfuran (2-MF) through furfuryl alcohol as an intermediate in gas/liquid phase reactions or by reactive distillation. ${ }^{23-25}$ Many studies have reported production of long chain alkanes from 2-MF via oxygenated intermediates. ${ }^{26,27}$ For instance, catalytic trimerisation of 2-MF was carried out in the presence of $\mathrm{H}_{2} \mathrm{SO}_{4}$ via formation of the ring-opened intermediate 4-oxopentanal followed by subsequent alkylation with two other molecules of 2-MF to yield a $\mathrm{C}_{15}$ oxygenate 5,5-bis(5-methylfuran-2-yl)pentan-2-one, which finally underwent HDO to form a saturated hydrocarbon diesel fraction. ${ }^{26}$ In an alternative approach, various diesel or jet fuel range branched alkanes were obtained by the integration of alkylation of 2-MF with different biomass-derived aldehydes or ketones (e.g., butanal, furfural, 5-methylfurfural, acetone, hydroxyacetone, cyclopentanone, and mesityl oxide) over acid catalysts and subsequent HDO catalyzed by supported metals. ${ }^{28-31}$ However, the major byproduct water generated in the alkylation reaction may adversely influence the interaction between the catalyst and the hydrophobic reactants. Based on this view point, a catalyst having an appropriate acidity along with hydrophobicity might facilitate the condensation reaction towards the targeted product.

In this work, alkylation of 2-MF with formalin - a commercially available $37 \%$ aqueous solution of formaldehyde (FA) - have been applied to generate the $C_{11}$ oxygenate bis(5-methylfuran-2-yl)methane (BMFM) with a series of sulfonic acid functionalized ionic liquids (AILs) and SBA-15 supported acidic ionic liquid catalysts (acidic SILCs) (Scheme 1). Parameters such as reaction time, temperature and catalyst loading were optimized to obtain high BMFM yield, and catalyst activity and selectivity were compared with commercially available sulfonic acid functionalized resins. Recyclability of an acidic SILC catalyst was confirmed by performing five 
consecutive reaction runs with intermediate phase-separation of the formed BMFM product after addition of $n$-heptane and water to the reaction mixture. The reactivity of other biomass-derived substrates, e.g. 3,4-dimethoxybenzaldehyde, furfural, glyceraldehyde and glycolaldehyde, with 2-MF in the catalytic systems have also been investigated.

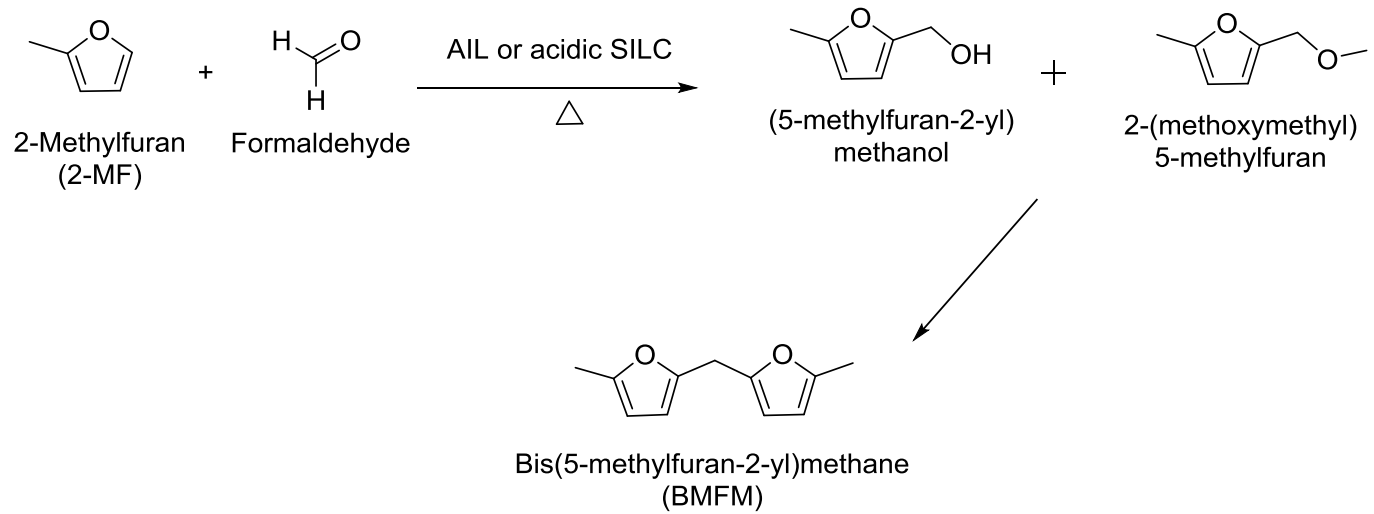

Scheme 1. Acidic ionic liquid (AIL) and supported acidic ionic liquid (acidic SILC) catalyzed alkylation of 2-MF and formalin.

\section{Experimental}

\section{Chemicals}

2-Methylfuran (2-MF, 99\%), formalin (37\% formaldehyde in water with 10-15\% methanol as stabilizer), glycolaldehyde (99\%), glyceraldehyde (95\%), 3,4-dimethoxybenzaldehyde (99\%), furfural ( $\geq 99 \%$ ), sodium hydrogen sulfate (ACS reagent), 1-methylimidazolium hydrogensulfate $\quad\left([\mathrm{MIM}] \mathrm{HSO}_{4}, \quad \mathbf{1 a}, \quad \geq 95 \%\right)$, 1-methylimidazolium chloride ([MIM]Cl, 1b, $\geq 95 \%)$, 1-butyl-3-methylimidazolium hydrogensulfate $\quad\left([\mathrm{BMIM}] \mathrm{HSO}_{4}, \quad \mathbf{2 a}, \quad \geq 94.5 \%\right), \quad$ 1-butyl-3-methylimidazolium methanesulfonate ([BMIM] $] \mathrm{CH}_{3} \mathrm{SO}_{3}, \mathbf{2 b}, \geq 95 \%$ ), Amberlyst-15 (hydrogen ion form, wet), DOWEX 50WX8-100 (hydrogen ion form, 50-100 mesh), and Nafion NR50 (hydrogen ion form) were purchased from Sigma-Aldrich and used as received.

Bis(5-methylfuran-2-yl)methane (BMFM) was isolated from the reaction mixture of reaction between 2-methylfuran and formalin over acidic SILC 4 (see below) by 
column chromatography (A60 silica gel, petroleum ether:ethyl acetate $=5: 1$ ), and the identity of the compound confirmed by NMR (Bruker AM360 NMR spectrometer) with $\mathrm{CD}_{3} \mathrm{OD}$ as solvent and the impurities were found to be insignificant. ${ }^{1} \mathrm{H}$ and ${ }^{13} \mathrm{C}$ NMR spectra are shown in ESI Figure S1.

\section{Preparation of catalysts}

1-Methyl-3-(4-sulfobutyl)imidazolium hydrogensulfate $\left(\left[\mathrm{BMIM}^{-} \mathrm{SO}_{3} \mathrm{H}\right] \mathrm{HSO}_{4}\right.$, AIL 3a) and 1-methyl-3-(4-sulfobutyl)imidazolium methanesulfonate ([BMIM- $\left.\mathrm{SO}_{3} \mathrm{H}\right] \mathrm{CH}_{3} \mathrm{SO}_{3}$, AIL 3b) were synthesized according to reported procedures. ${ }^{32-33}$ SBA-15 supported acidic ionic liquids (acidic SILCs) 4 and 5 were also prepared by post-grafting of the corresponding $\mathrm{SO}_{3} \mathrm{H}$ functionalized ionic liquids onto the mesoporous solid support, as demonstrated previously. ${ }^{34-36}$

\section{Catalyst characterization}

Powder X-ray diffraction (XRD) patterns of the catalysts were obtained on a Bruker D8 Advance diffractometer (Germany) using $\mathrm{CuK} \alpha$ radiation $(\lambda=0.154 \mathrm{~nm})$. The diffractograms were recorded in the $2 \theta$ range of $0.2-5.0^{\circ}$ at the rate of $0.1 / \mathrm{min}$ $(40 \mathrm{kV}, 20 \mathrm{~mA})$.

The surface area of the acidic SILC and parent SBA-15 materials was measured by nitrogen sorption studies. Nitrogen adsorption/desorption isotherms were collected at $-196{ }^{\circ} \mathrm{C}$ on a Micromeritics ASAP 2020. Before the analysis, the samples were degassed at $90{ }^{\circ} \mathrm{C}$ under vacuum for $3 \mathrm{~h}$. The surface area was calculated using the BET model and the pore size distributions were calculated from $\mathrm{BJH}$ adsorption branches.

The acid strength of the acidic SILCs was measured by the Hammett acidity function $\left(H_{0}\right)$ using various Hammett indicators ( $p$-nitroaniline: $\mathrm{pK}_{\mathrm{a}}=0.99$, o-nitroaniline: $\quad \mathrm{pK}_{\mathrm{a}}=-0.29, \quad$ 4-chloro-2-nitroaniline: $\mathrm{pK}_{\mathrm{a}}=-1.03$, 2,4-dichloro-6-nitroaniline: $\mathrm{pK}_{\mathrm{a}}=-3.32,2$,4-dinitroaniline: $\mathrm{pK}_{\mathrm{a}}=-4.53$, benzalacetophenone: $\mathrm{pK}_{\mathrm{a}}=-5.6$, anthraquinone: $\mathrm{pK}_{\mathrm{a}}=-8.2,2,4,6$-trinitroaniline: $\mathrm{pK}_{\mathrm{a}}$ $=-10.10, p$-nitrotoluene: $\mathrm{pK}_{\mathrm{a}}=-11.35, p$-nitrochlorobenzene: $\mathrm{pK}_{\mathrm{a}}=-12.70$ ) by 
following previous reports. ${ }^{37,38}$ The acidic SILCs $(50 \mathrm{mg})$ were dried in vacuum at 80 ${ }^{\circ} \mathrm{C}$ for $6 \mathrm{~h}$ prior to the indicator test, before being transferred to a test tube containing $1 \mathrm{~mL}$ of toluene and $0.1 \%$ solution of indicator (3-5 drops). The $H_{0}$ values of the acidic SILCs were obtained on the basis of two adjacent pKa values of indicators (colourless $<H_{0}<$ appearance of colour).

\section{Alkylation reaction of 2-MF with formalin}

Alkylation reactions were carried out in Ace pressure tubes equipped with a magnetic stirring bar and placed in an oil bath. Typically, a reaction solution containing $0.82 \mathrm{~g}$ 2-MF (10 mmol), $0.405 \mathrm{~g}$ formalin (5 mmol formaldehyde) or other aldehyde and $5 \mathrm{~mol} \%$ or $50 \mathrm{mg}$ catalyst was stirred at a set temperature for a specific reaction time. Upon completion, the reaction mixture was diluted with water $(5 \mathrm{~mL})$ and extracted with $n$-heptane $(3 \times 5 \mathrm{~mL})$. Identification of the products were confirmed by GC-MS (Agilent 6850 GC system coupled with an Agilent 5975C mass detector), and the conversions of 2-MF and yields of BMFM and other oxygenates were quantified by GC-FID analysis (Agilent 6890N instrument, HP-5 capillary column $30.0 \mathrm{~m} \times 320 \mu \mathrm{m} \times 0.25 \mu \mathrm{m}$ ) by making series of standards of $2-\mathrm{MF}$ and BMFM with naphthalene as internal standard. The conversion of 2-MF and the yield and selectivity of BMFM were calculated according to equations (1)-(3) taking into account that only $0.5 \mathrm{~mol}$ of BMFM can be formed per mol of 2-MF.

$$
\begin{aligned}
& \text { Conversion of 2-MF (\%): } X=\frac{n_{2-M F}^{t}}{n_{2-M F}^{i}} \times 100 \\
& \text { BMFM yield (\%): } Y=\frac{n_{B M F M}^{\mathrm{t}}}{n_{\mathrm{2}-M F}^{i}} \times 100 \\
& \text { BMFM selectivity }(\%): S=\frac{Y}{X} \times 100
\end{aligned}
$$

\section{Results and Discussion}

Application of AILs and acidic SILCs for the conversion of biomass-derived sugars to high value chemicals, e.g. alkyl levulinate, are previously well documented 
by our group. ${ }^{39,40}$ Prompted by these biomass-related studies, a series of acidic and non-acidic ionic liquids, and acidic SILCs (Figure 1) were employed for the alkylation reaction of 2-MF and formalin.
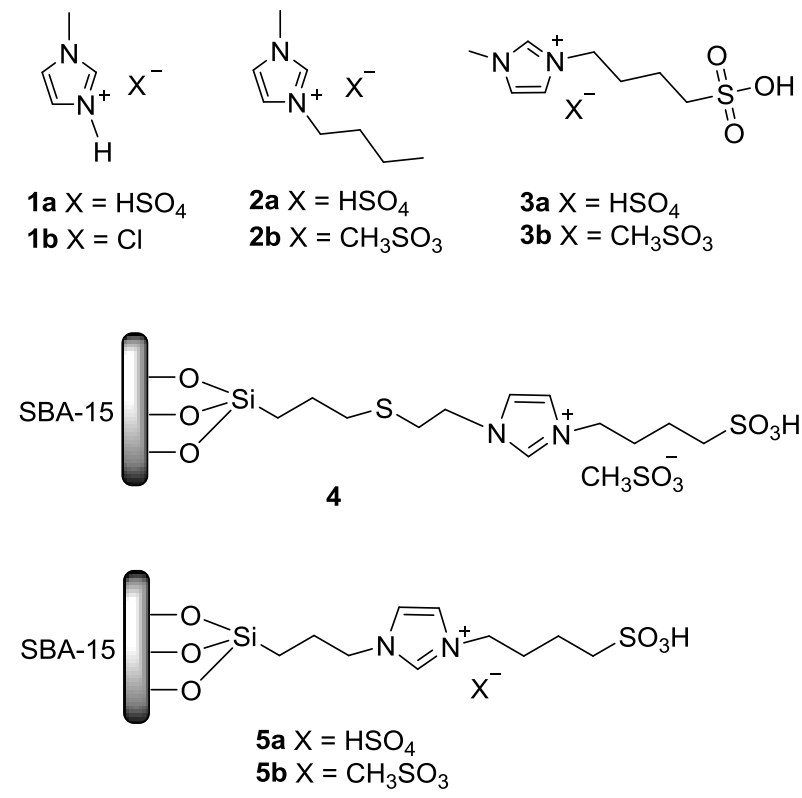

Figure 1. Applied acidic and non-acidic ionic liquids (AILs and non-AILs) and SBA-15 supported AILs (acidic SILCs).

The XRD patterns of the prepared acidic SILC materials are shown in Figure S3. A strong intense and characteristic peak of the (100) plane appeared at $2 \theta$ value of $0.9^{\circ}$ corresponding to $\mathrm{d}_{100}=9.7 \mathrm{~nm}$ (calculated from Bragg's Law), implying the presence of two-dimensional hexagonal space symmetry, p6mm, with ordered hexagonal array of one-dimensional mesoporous channel structure. Other characteristic peaks of the (110) and (200) planes, supposed to appear at $2 \theta$ value of $1.5-2^{o}{ }^{36}$ seemed to be diminished. The disappearance of these planes was possibly caused by the anchoring of bulky and large amount of functionalized ionic liquids on the pore walls of SBA-15.

The nitrogen sorption isotherms and textual properties of the solid materials are further given in Figure S3 and Table 1, respectively. The sorption isotherms of the acidic SILCs indicated type IV isotherms with H1 hysteresis loops. The steep increase observed at relative pressure $\mathrm{P} / \mathrm{P}_{0}$ around 6.0-8.0 is analogous to what previously has 
been reported for functionalized materials. ${ }^{36}$ The surface areas of the acidic SILCs were drastically decreased from $727 \mathrm{~m}^{2} / \mathrm{g}$ in pristine SBA-15 to 153,57 and $51 \mathrm{~m}^{2} / \mathrm{g}$ for acidic SILC $\mathbf{4}, \mathbf{5 a}$ and $\mathbf{5 b}$, respectively, due to anchoring of the functionalized ionic liquids on the pore walls.

The alkylation reactions of 2-MF and formalin were carried out at $40{ }^{\circ} \mathrm{C}$ for $2 \mathrm{~h}$ and the results are presented in Figure 2. Experiments were initially conducted with ILs having no $\mathrm{SO}_{3} \mathrm{H}$ group to confirm the essential role of strong acid sites in the reaction. The ILs with $\mathrm{Cl}^{-}$and $\mathrm{CH}_{3} \mathrm{SO}_{3}{ }^{-}$anions (1b and $\mathbf{2 b}$ ) yielded less than $1 \%$ of BMFM (Figure 2a). A significant improvement in BMFM yield to 20 and $30 \%$ was observed when the anions were replaced with $\mathrm{HSO}_{4}{ }^{-}$in the corresponding ILs $\mathbf{1 a}$ and 2a, respectively. However, when the strongly acidic $\mathrm{SO}_{3} \mathrm{H}$ group was introduced into the AILs 3a ([BMIM-SO $\left.\left.{ }_{3} \mathrm{H}\right] \mathrm{HSO}_{4}\right)$ and 3b ([BMIM-SO$\left.\left.{ }_{3} \mathrm{H}\right] \mathrm{CH}_{3} \mathrm{SO}_{3}\right)$, the yield of BMFM was drastically enhanced to 67 and $65 \%$, respectively, substantiating that strong Brønsted acid sites are imperative to efficiently catalyze the reaction. Even though a high yield of BMFM can be achieved with the AILs, such homogeneous systems suffer from tedious work-up for separation of the reaction mixture and the catalyst.

Introduction of sulfonic acid groups onto a solid support, such as SBA-15, by covalently anchoring is attractive due to the obtained properties, e.g. porosity, high thermal stability, high surface area, easy recoverability and reuse. Testing of acidic SILC 4 for the alkylation reaction under similar reaction conditions as for the AILs resulted in a BMFM yield of $30 \%$. This yield was much lower than the yields obtained with the neat $\mathrm{SO}_{3} \mathrm{H}$ AILs $\mathbf{3 a}$ and $\mathbf{3} \mathbf{b}$, but comparable with the commercially available solid acid resin catalyst Amberlyst-15 and better than Nafion and Dowex (Figure 2a). Interestingly, a significantly higher selectivity to BMFM (90\%) was found with acidic SILC 4 than with the $\mathrm{SO}_{3} \mathrm{H}$-resins (60-70\%). The relatively good performance of acidic SILC 4 was probably not only due to the presence of strong $\mathrm{SO}_{3} \mathrm{H}$ groups but also related to the narrow pore size distribution $(52 \AA$, Table $\mathrm{S} 1)$ of 
SBA-15, which can accommodate bulky molecules and alleviate diffusion limitations. Moreover, the higher selectivity towards BMFM with acidic SILC 4 compared with neat $\mathrm{SO}_{3} \mathrm{H}$-resins might be due to the presence of mesoporous channels along with relatively high surface area of the support, as confirmed by the XRD and $\mathrm{N}_{2}$ sorption studies (Figure S3 and S4 and Table S1).

To compare the activity of the acidic SILC 4 towards BMFM formation against the other applied catalysts, turn over numbers (TONs) and turn over frequencies (TOFs) were calculated based on the acid sites present in the catalysts. The acid density of acidic SILC 4 was measured to $1.10 \mathrm{mmol} / \mathrm{g}$ by titrating against diluted $\mathrm{NaOH}$ with phenolphthalein as indicator as done in other studies. ${ }^{41,42}$ As illustrated in Figure 2b, the acidic SILC 4 exhibited the highest efficiency based on TOF (13.5 $\left.\mathrm{h}^{-1}\right)$ and TON (27) among the catalysts employed. In all experiments, intermediates such as, e.g. (5-methylfuran-2-yl)methanol and 2-(methoxymethyl)-5-methylfuran (structures shown in Scheme 1) were identified by GC-MS and found to be formed in totally 1-5\% yield. Moreover, several undesired (unidentified) products were also observed by GC-MS.

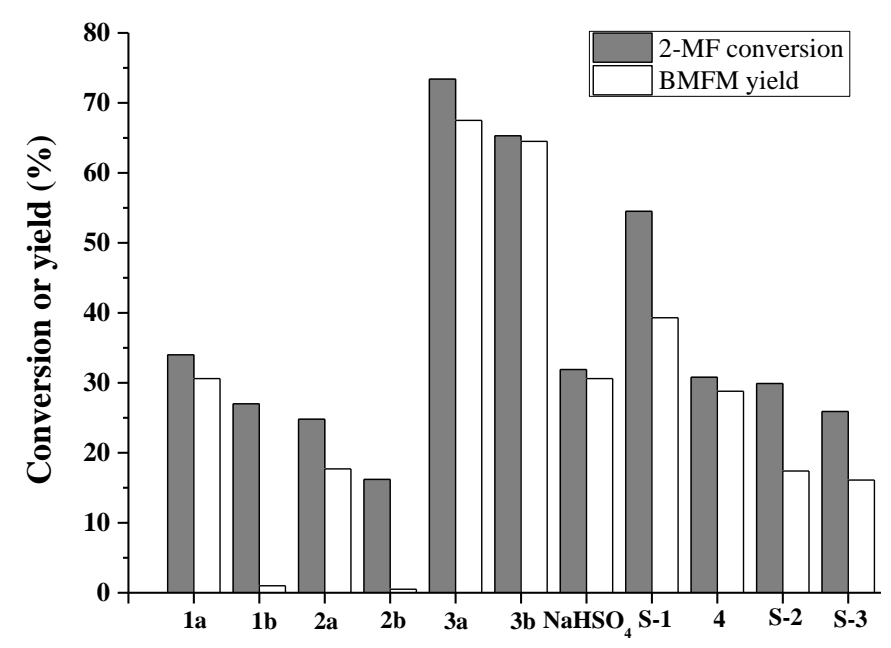

Figure 2a. Alkylation reaction of 2-MF with formalin using various catalysts. Reaction conditions: $0.82 \mathrm{~g}$ 2-MF (10 mmol), $0.405 \mathrm{~g}$ formalin $\left(5 \mathrm{mmol}\right.$ formaldehyde), $5 \mathrm{~mol} \%$ or $50 \mathrm{mg}$ catalyst, $40^{\circ} \mathrm{C}, 2$ h (1a: [MIM] $\mathrm{HSO}_{4} ; \mathbf{1 b}$ : [MIM]Cl; 2a: [BMIM] $\mathrm{HSO}_{4} ; \mathbf{2 b}$ : [BMIM] $\mathrm{CH}_{3} \mathrm{SO}_{3} ; \mathbf{3 a}$ : [BMIM-SO $\mathrm{BH}_{3} \mathrm{HSO}_{4}$; 3b: [BMIM- $\left.\mathrm{SO}_{3} \mathrm{H}\right] \mathrm{CH}_{3} \mathrm{SO}_{3}$; S-1: Amberlyst; S-2: DOWEX 50WX8-100; S-3: Nafion NR 50). 


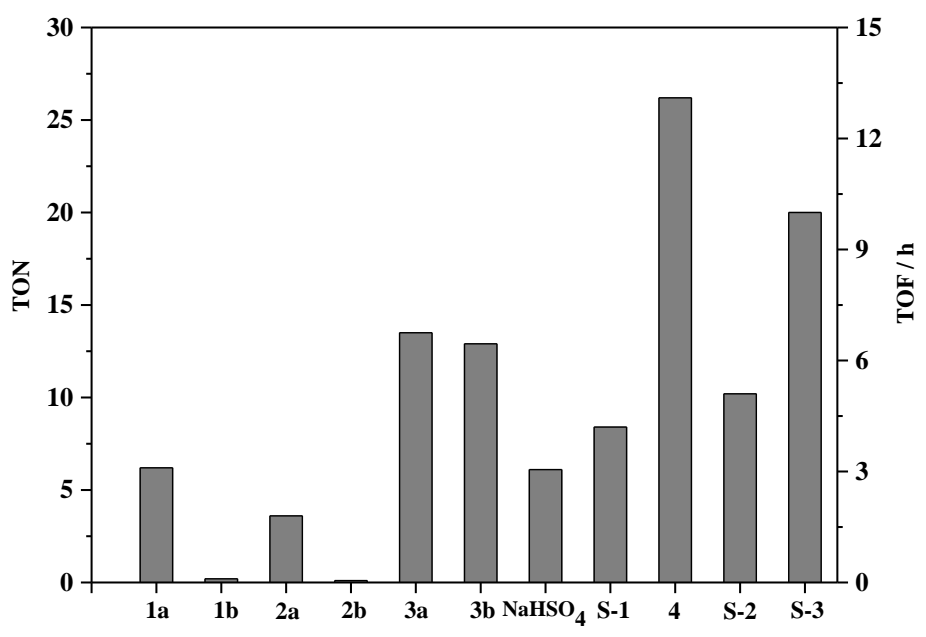

Figure 2b. TONs and TOFs of catalysts towards the formation of BMFM. Reaction conditions: $0.82 \mathrm{~g}$ 2-MF (10 mmol), $0.405 \mathrm{~g}$ formalin ( $5 \mathrm{mmol}$ formaldehyde), $5 \mathrm{~mol} \%$ or $50 \mathrm{mg}$ catalyst, $40{ }^{\circ} \mathrm{C}, 2 \mathrm{~h}(\mathbf{1 a}$ : [MIM] $\mathrm{HSO}_{4} ; \mathbf{1 b}$ : [MIM]Cl; 2a: [BMIM] $\mathrm{HSO}_{4} ; \mathbf{2 b}:\left[\mathrm{BMIM}^{2} \mathrm{CH}_{3} \mathrm{SO}_{3} ; \mathbf{3 a}:\left[\mathrm{BMIM}_{-} \mathrm{SO}_{3} \mathrm{H}\right] \mathrm{HSO}_{4} ; \mathbf{3 b}\right.$ : [BMIM- $\left.\mathrm{SO}_{3} \mathrm{H}\right] \mathrm{CH}_{3} \mathrm{SO}_{3}$; S-1: Amberlyst; S-2: DOWEX 50WX8-100; S-3: Nafion NR 50).

The influence of reaction temperature and time on the conversion of 2-MF and BMFM yield was also studied with Amberlyst-15 and acidic SILC 4, and the results are compiled in Figure 3. As mentioned above, the catalytic activity of Amberlyst-15 was at $40{ }^{\circ} \mathrm{C}$ higher than that of acidic SILC 4, but the selectivity was lower. At $60{ }^{\circ} \mathrm{C}$ both 2-MF conversions and BMFM yields were increased with the catalysts (as expected), and interestingly acidic SILC 4 gave a slightly higher BMFM yield (76\%) after $4 \mathrm{~h}$ reaction than Amberlyst-15 (71\%). When the reaction time was prolonged to $8 \mathrm{~h}$, the yield of BMFM increased further to above $80 \%$ in the presence of the acidic SILC 4. In contrast, the BMFM yield stagnated at higher temperature, which was also observed to some extent in the case of acidic SILC 4 when the reaction temperature was increased to $80{ }^{\circ} \mathrm{C}$. Formation of possible trimerized product of 2-MF, i.e. 5,5-bis(5-methylfuran-2-yl)-pentan-2-one, might be responsible for the lower yield of BMFM observed at relatively high temperature, as previously reported. ${ }^{26,27}$ In all reactions BMFM was the major product along with minor amount of the intermediate, such as (5-methyl-2-furyl)methanol and 2-(methoxymethyl)-5-methylfuran, which were identified in the biphasic catalytic systems (see ESI Figure S2). 

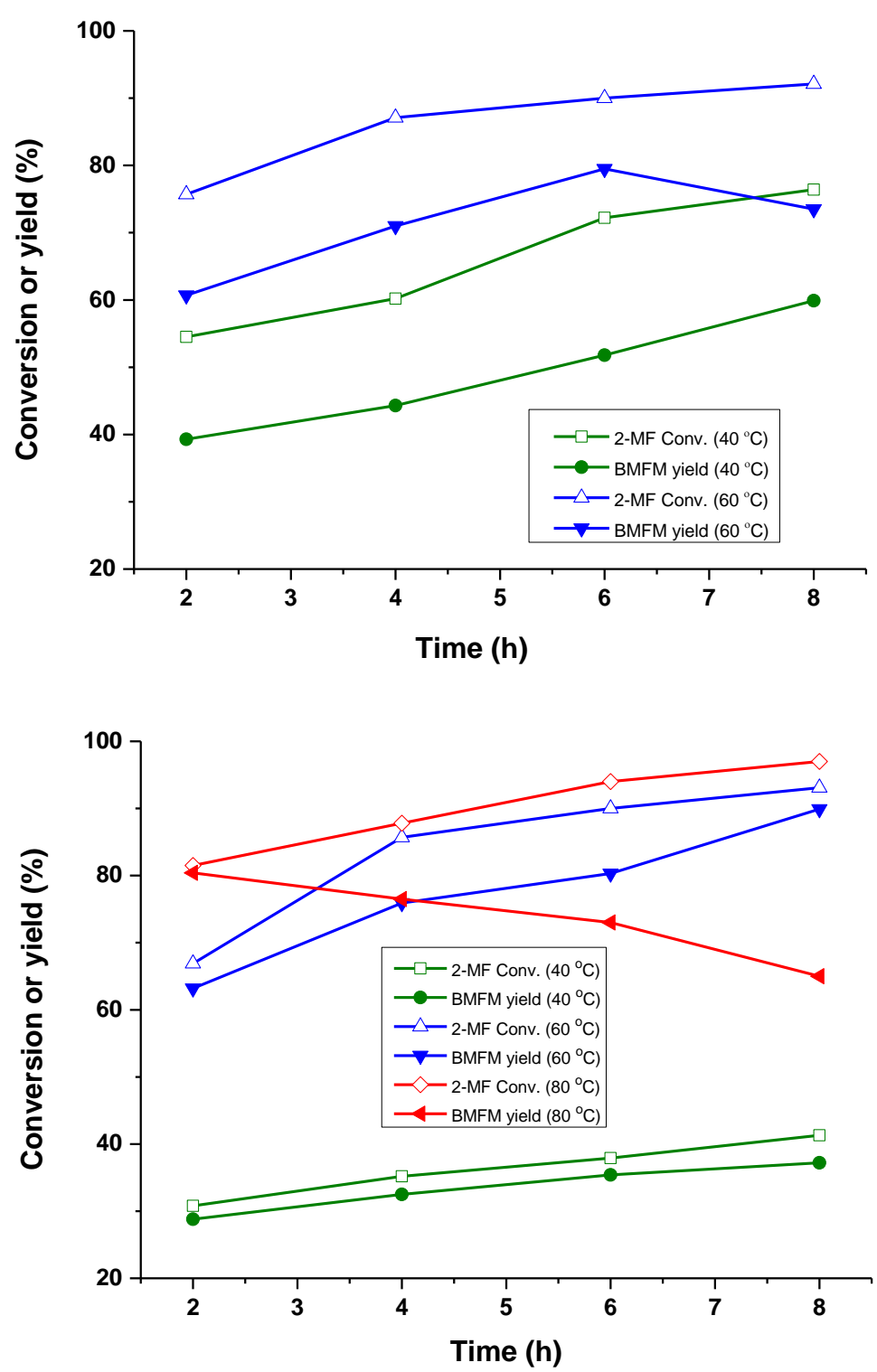

Figure 3. Effect of reaction temperature and time on the alkylation of 2-MF with formalin catalyzed by Amberlyst-15 (top) and acidic SILC 4 (bottom). Reaction conditions: 0.82 g 2-MF(10 mmol), 0.405 g formalin ( $5 \mathrm{mmol}$ formaldehyde), $50 \mathrm{mg}$ catalyst.

To further investigate the influence of the anchored AIL moiety of SBA-15 on catalytic activity in the alkylation reaction, alternative SBA-15 supported AILs (acidic SILC 5a and 5b) (Figure 1) were also synthesized ${ }^{34-36}$ and tested. As shown in Table 1, no significant difference between these SILCs was observed with respect to conversion of 2-MF (66-70\%) and BMFM yield (60-63\%) initially during reaction (2 h). However, as reaction time progressed (4-8 h) SILC 4 - with a relatively long alkyl chain - demonstrated to be more catalytically active than SILCs $\mathbf{5 a}$ and $\mathbf{5 b}$. Results 
from the nitrogen sorption studies revealed the surface areas of acidic SILCs 5a and 5b to be almost three-fold lower than SILC 4 (vide supra). This suggests that the ionic liquids anchored in the mesoporous walls of the former SILCs in particular induced pore blocking, which would contribute to hampered transport of the bulky molecules and result in lower yield of BMFM at longer reaction time. In addition, SILC $\mathbf{5 b}$ bearing a $\mathrm{CH}_{3} \mathrm{SO}_{3}{ }^{-}$anion gave slightly higher yields of BMFM (81\%) than the corresponding SILC 5a with $\mathrm{HSO}_{4}{ }^{-}$anion (76\%).

Table 1. Alkylation of 2-MF with formalin using acidic SILCs

\begin{tabular}{cccccc}
\hline SILC & $\begin{array}{c}\text { Time } \\
(\mathrm{h})\end{array}$ & $\begin{array}{c}\text { Conversion of 2-MF } \\
(\%)\end{array}$ & $\begin{array}{c}\text { Yield of BMFM } \\
(\%)\end{array}$ & TON & $\begin{array}{c}\text { TOF } \\
\left(\mathrm{h}^{-1}\right)\end{array}$ \\
\hline $\mathbf{4}$ & 2 & 67 & 63 & 28 & 14 \\
& 4 & 86 & 76 & 34 & 9 \\
& 8 & 92 & 88 & 40 & 5 \\
$5 \mathbf{5 a}$ & 2 & 70 & 60 & 20 & 10 \\
& 4 & 85 & 72 & 24 & 6 \\
& 8 & 86 & 76 & 25 & 3 \\
$5 \mathbf{5 b}$ & 2 & 66 & 61 & 25 & 13 \\
& 4 & 76 & 75 & 31 & 8 \\
& 8 & 81 & 81 & 33 & 4 \\
\hline
\end{tabular}

Reaction conditions: $0.82 \mathrm{~g}$ 2-MF ( $10 \mathrm{mmol}), 0.405 \mathrm{~g}$ formalin $(5 \mathrm{mmol}$ formaldehyde), $50 \mathrm{mg}$ catalyst, $60^{\circ} \mathrm{C}$.

The acid density of the acidic SILCs $\mathbf{5 a}$ and $\mathbf{5 b}$ was measured (by the same procedure used for acidic SILC 4) to be 1.51 and $1.22 \mathrm{mmol} / \mathrm{g}$, respectively. A comparison between TONs and TOFs of the acidic SILCs 4, 5a and $\mathbf{5 b}$ after normalizing by the acid density clearly showed that the order of activity of the three acidic SILCs were: $\mathbf{4}>\mathbf{5 b}>\mathbf{5 a}$ (Table 1). However, these results do not reveal the role of acid strength on the yield of product. The acid strength of the examined catalysts was expressed by the Hammett acidity function $\left(H_{0}\right)$, which was scaled by 
the pKa values of the indicators. Using this method the order of acid strength was found to be: acidic SILC $4\left(-5.6<H_{0}<-4.53\right)>\mathbf{5 a}\left(-4.53<H_{0}<-3.32\right) \approx \mathbf{5 b}(-4.53<$ $H_{0}<-3.32$ ). These results substantiate that the higher catalytic activity (i.e. higher BMFM yield) found for acidic SILC 4 was related to its stronger acidity and not to the number of acid sites which was lower than for acidic SILCs $\mathbf{5 a}$ and $\mathbf{5 b}$.

After establishing that acidic SILC 4 was the most active of the supported catalysts, the influence of catalyst loading in the alkylation reaction was studied using this catalyst. The results obtained after $6 \mathrm{~h}$ of reaction are compiled in Table 2. As the amount of added acidic SILC 4 was increased from 10 to $50 \mathrm{mg}$, the yield of BMFM improved from 62 to $80 \%$. With higher catalyst loading of $75 \mathrm{mg}$ the yield of BMFM decreased to $61 \%$. In line with this, the TON and TOF reached a maximum of 281 and $47 \mathrm{~h}^{-1}$, respectively, with $10 \mathrm{mg}$ catalyst after $6 \mathrm{~h}$ of reaction, while higher catalyst loading gave lower catalyst activity. After $20 \mathrm{~h}$ of reaction, the yield of BMFM increased from 62 to $87 \%$ in the $10 \mathrm{mg}$ experiment corresponding to a lower TOF of $19 \mathrm{~h}^{-1}$ and a TON of 397. On the other hand, when the reaction time was extended from 6 to $20 \mathrm{~h}$ for the experiment with $75 \mathrm{mg}$ of catalyst, the yield of BMFM decreased gradually to $57 \%$. This decrease was most likely due to formation of byproducts (unidentified) formed by, e.g. acid-catalyzed hydrolysis of the furanics. Based on the studies it is apparent, that application of a low amount of catalyst is preferable to obtain high catalyst TON and TOF as well as high BMFM product selectivity at longer reaction time.

Table 2. Effect of loading of acidic SILC 4 on the alkylation of 2-MF with formalin

\begin{tabular}{ccccc}
\hline $\begin{array}{c}\text { Catalyst loading } \\
(\mathrm{mg})\end{array}$ & $\begin{array}{c}\text { Conversion of 2-MF } \\
(\%)\end{array}$ & $\begin{array}{c}\text { Yield of BMFM } \\
(\%)\end{array}$ & TON & $\begin{array}{c}\text { TOF } \\
\left(\mathrm{h}^{-1}\right)\end{array}$ \\
\hline 10 & 68 & 62 & 281 & 47 \\
25 & 80 & 72 & 130 & 22 \\
50 & 90 & 80 & 72 & 12 \\
75 & 91 & 61 & 37 & 6 \\
\hline
\end{tabular}

Reaction conditions: $0.82 \mathrm{~g}$ 2-MF (10 mmol), $0.405 \mathrm{~g}$ formalin $(5 \mathrm{mmol}$ formaldehyde $), 60^{\circ} \mathrm{C}, 6 \mathrm{~h}$. 
The recyclability of the acidic SILC 4 catalyst was further studied in five consecutive alkylation reaction cycles $\left(60^{\circ} \mathrm{C}, 4 \mathrm{~h}\right)$ as shown in Figure 4 . After each cycle, the product was extracted from the reaction mixture with the non-polar, organic solvent $n$-heptane after dilution with water. Subsequently, the catalyst was separated by filtration, washed with acetone $(3 \times 20 \mathrm{~mL})$ and dried at $110{ }^{\circ} \mathrm{C}$ for $6 \mathrm{~h}$. The obtained catalyst was directly used for the next run. In the first four consecutive catalytic runs, the BMFM yield remained rather constant around $70-75 \%$ with $2-\mathrm{MF}$ conversion of $80-85 \%$ ( $86-94 \%$ selectivity). A slight decrease in product yield to $63 \%$ (72\% 2-MF conversion) was observed after the fifth reaction run, possibly due to impeding of the active sites by adsorbed organic moieties and water coming from formalin, which were not removed by the simple washing and drying procedure. Importantly, the selectivity to BMFM remained, however, unchanged even after the fifth run.

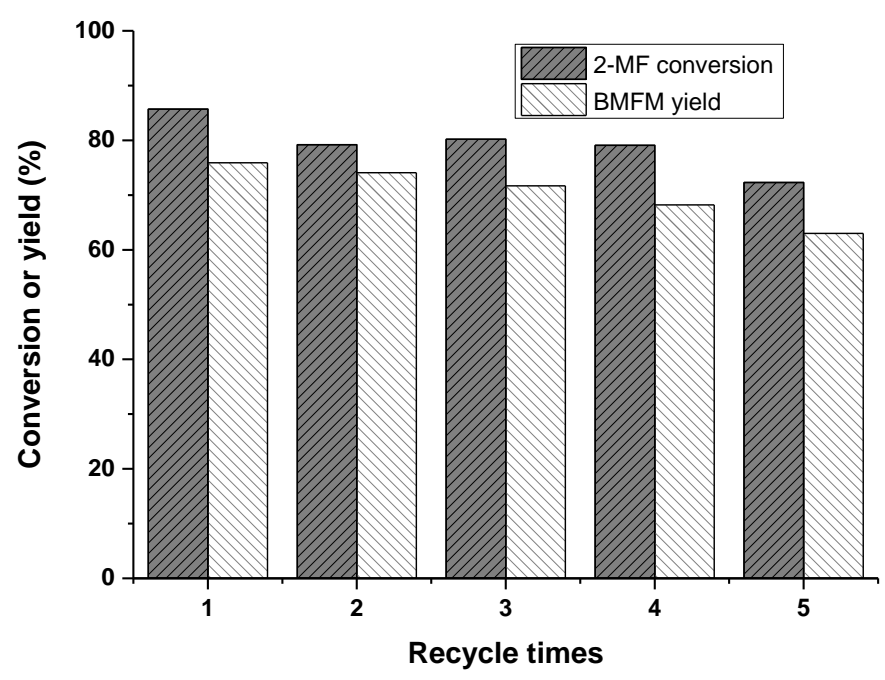

Figure 4. Recyclability of acidic SILC 4 in alkylation reaction of 2-MF and formalin. Reaction conditions: $0.82 \mathrm{~g}$ 2-MF (10 mmol), $0.405 \mathrm{~g}$ formalin $\left(5 \mathrm{mmol}\right.$ formaldehyde), $50 \mathrm{mg}$ catalyst, $60^{\circ} \mathrm{C}, 4$ h.

To examine the deactivation in more detail, quantification of the acid density of acidic SILC 4 was performed after the fifth run (after washing with acetone and drying at $80{ }^{\circ} \mathrm{C}$ overnight) by titration against $\mathrm{NaOH}$ with phenolphthalein as 
indicator. The total number of acid sites was found to descend from the originally 1.10 to $0.83 \mathrm{mmol} / \mathrm{g}$, suggesting that strongly bound adsorbed molecules remained bound to the active sites despite the washing and drying procedure. Moreover, the acid strength had decreased from $-5.6<H_{0}<-4.53$ to $-4.53<H_{0}<-3.32$, indicating that some of the strong acid sites could be hampered by water molecules.

The alkylation reaction protocol using acidic SILC $\mathbf{4}$ was also extended to other biomass-derived aldehyde substrates like, e.g. 3,4-dimethoxybenzaldehyde, furfural, glycolaldehyde and glyceraldehyde, and the results are summarized in Table 3 . All the substrates, except glyceraldehyde, efficiently reacted with 2-MF using acidic SILC 4 yielding $78-87 \%$ of the corresponding condensed product. As mentioned before, the high yields of products could be achieved not only due to the presence of strong acid sites, but also due to presence of well-ordered mesoporous channels, a large pore size distribution (around $50 \AA$ ) and relatively high surface area of the SBA-15 material (Figure S3 and Table S1), which facilitate transport of the bulky molecules in the support. The electron-withdrawing effect of a $\alpha$-hydroxy group next to the formyl group in substrates has been reported to facilitate high yields in the alkylation of the aldehyde with $2-\mathrm{MF}^{29}$ In line with this, a high yield (87\%) of glycolaldehyde and 2-MF condensed product was obtained. However, having an additional $\beta$-hydroxy group in the substrate (glyceraldehyde) apparently led to the formation of the corresponding product in low yield (35\%), most likely due to consecutive byproduct formation by, e.g. dehydration, condensation etc. (byproducts not identified). 
Table 3. Alkylation of 2-MF with biomass-derived aldehydes using acidic SILC 4

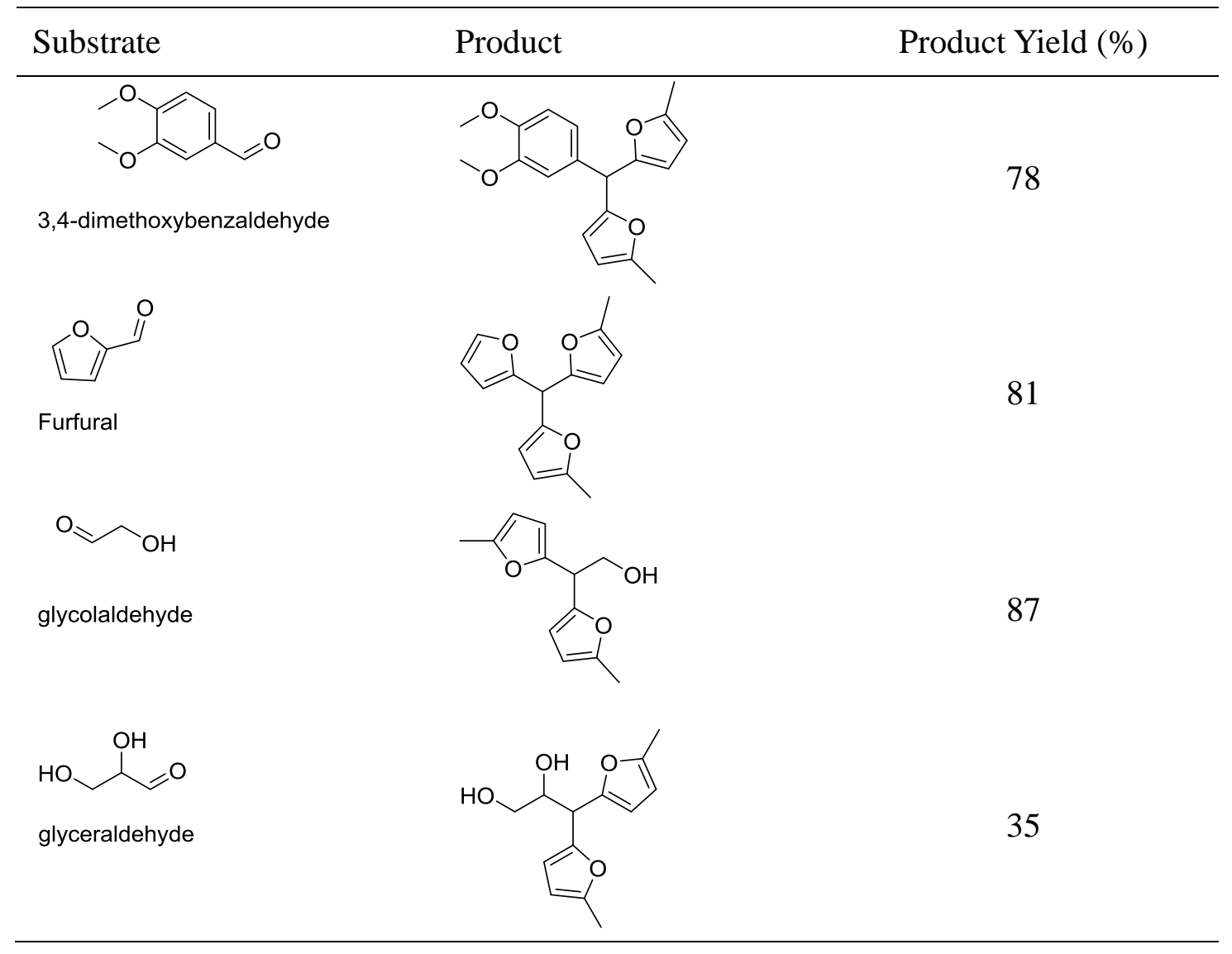

Reaction conditions: $0.82 \mathrm{~g}$ 2-MF (10 mmol), $5 \mathrm{mmol}$ substrate (in case of glyceraldehyde and 3,4-dimethoxybenzaldehyde, $0.3 \mathrm{~g}$ of methanol was added to have a clear reaction mixture before adding catalyst), $60^{\circ} \mathrm{C}, 4 \mathrm{~h}$.

\section{Conclusions}

A biphasic reaction protocol has been established for efficient alkylation of 2-MF with formalin to form BMFM using a number of acidic ionic liquid (AIL) catalysts. Mesoporous SBA-15 supported AIL (acidic SILC 4) was found to have the highest catalytic activity among the catalysts employed, which might be ascribed to its high acid strength, large pore size and suitable hydrophobic properties. The highest TON (281) and TOF $\left(47 \mathrm{~h}^{-1}\right)$ towards the formation of BMFM was obtained with $10 \mathrm{mg}$ of acidic SILC 4 with a selectivity of above 90\%. The acidic SILC 4 catalyst could be readily recovered from the reaction mixture by filtration, and reused for at least five consecutive runs with a rather constant BMFM yield of around 70\%. Moreover, under 
the optimized reaction conditions, alkylation of 2-MF with other biomass-derived aldehydes was also achieved with $80 \%$ or higher yield of the corresponding furanic products.

\section{Supporting Information}

${ }^{1} \mathrm{H}$ and ${ }^{13} \mathrm{C}$ NMR spectra of BMFM, pictures of phase separation of alkylation reaction mixture, $\mathrm{N}_{2}$ sorption isotherms, $\mathrm{XRD}$ patterns and physicochemical properties of SILCs.

\section{Acknowledgements}

We thank the Danish Council for Independent Research-Technology and Production Sciences (No.10-081991), and Chinese State Scholarship Fund (No. 201306670004) for financial support of the work.

\section{References}

(1) Himmel, G. W.; Ding, S.-Y.; Johnson, D. K.; Adney, W. S.; Nimlos, M. R.; Brady, J. W.; Foust, T. D. Biomass recalcitrance: Engineering plants and enzymes for biofuels production. Science 2007, 315, 804-807.

(2) Corma, A.; Iborra, S.; Velty, A. Chemical routes for the transformation of biomass into chemicals. Chem. Rev. 2007, 107, 2411-2502.

(3) Saravanamurugan, S.; Paniagua, M.; Melero, Juan A.; Riisager, A. Efficient isomerization of glucose to fructose over zeolites in consecutive reactions in alcohol and aqueous media. J. Am. Chem. Soc. 2013, 135, 5246-5249.

(4) Wettstein, S. G.; Alonso, D. M.; Chonga, Y.; Dumesic, J. A. Production of levulinic acid and gamma-valerolactone (GVL) from cellulose using GVL as a solvent in biphasic systems. Energy Environ. Sci. 2012, 5, 8199-8203

(5) Kunkes, E. L.; Simonetti, D. A.; West, R. M.; Serrano-Ruiz, J. C.; Gärtner, C. A.; Dumesic, J. A. Catalytic conversion of biomass to monofunctional hydrocarbons and targeted liquid-fuel classes. Science 2008, 322, 417-421.

(6) Corma, A.; Torre, O. de la.; Renz, M.; Villandier, N. Production of high-quality 
diesel from biomass waste products. Angew. Chem. Int. Ed. 2011, 50, 2375-2378.

(7) Serrano-Ruiz, J. C.; Pineda, A.; Balu, A. M.; Luquea, R.; Campeloa, J. M.; Romero, A. A.; Ramos-Fernández, J. M. Catalytic transformations of biomass-derived acids into advanced biofuels. Catal. Today 2012, 195, 162-168.

(8) West, R. M.; Liu, Z. Y.; Peter, M.; Dumesic, J. A. Liquid alkanes with targeted molecular weights from biomass-derived carbohydrates. ChemSusChem 2008, 1, 417-424.

(9) Bohre, A.; Dutta, S.; Saha, B.; Abu-Omar, Mahdi M. Upgrading furfurals to drop-in biofuels: An overview. ACS Sustain. Chem. Eng. 2015, 3, 1263-1277.

(10) Harvey, B. G.; Meylemans, H. A. The role of butanol in the development of sustainable fuel technologies. J. Chem. Technol. Biotechnol. 2011, 86, 2-9.

(11) Berteau, P.; Delmon, B.; Dallons, J. L.; Vangysel, A. Acid-base properties of silica-aluminas: Use of 1-butanol dehydration as a test reaction. Appl. Catal. 1991, 70, 307-323.

(12) Wright, M. E.; Harvey, B. G.; Quintana, R. L.; Highly efficient zirconium-catalyzed batch conversion of 1-butene: A new route to jet fuels. Energy Fuels 2008, 22, 3299-3302.

(13) Harvey, B. G.; Quintana, R. L. Synthesis of renewable jet and diesel fuels from 2-ethyl-1-hexene. Energy Environ. Sci. 2010, 3, 352-357.

(14) Subrahmanyam, A. V.; Thayumanavan, S.; Huber, G. W. C-C bond formation reactions for biomass-derived molecules. ChemSusChem 2010, 3, 1158-1161.

(15) Xu, W.; Liu, X.; Ren, J.; Liu, H.; Ma, Y.; Wang, Y.; Lu, G. Synthesis of nanosized mesoporous $\mathrm{Co}-\mathrm{Al}$ spinel and its application as solid base catalyst. Micropor. Mesopor. Mater. 2011, 142, 251-257.

(16) Zapata, P. A.; Faria, J.; Ruiz, M. P.; Resasco, D. E. Condensation/hydrogenation of biomass-derived oxygenates in water/oil emulsions stabilized by nanohybrid catalysts. Top. Catal. 2012, 55, 38-52.

(17) Crossley, S.; Faria, J.; Shen, M.; Daniel E. Resasco. Solid nanoparticles that catalyze biofuel upgrade reactions at the water/oil interface. Science 2010, 327, 68-70. (18) Xu, W.; Xia, Q.; Zhang, Y.; Guo, Y.; Wang Y.; Lu, G. Effective production of 
octane from biomass derivatives under mild conditions. ChemSusChem 2011, 4, $1758-1761$.

(19) Xia, Q.-N.; Cuan, Q.; Liu, X.-H.; Gong, X.-Q.; Lu, G.-Z.; Wang, Y.-Q. $\mathrm{Pd} / \mathrm{NbOPO}_{4}$ multifunctional catalyst for the direct production of liquid alkanes from aldol adducts of furans. Angew. Chem. Int. Ed. 2014, 53, 9755-9760.

(20) Huang, Y.-B.; Yang, Z.; Dai, J.-J.; Guo, Q.-X.; Fu, Y. Production of high quality fuels from lignocellulose-derived chemicals: A convenient $\mathrm{C}-\mathrm{C}$ bond formation of furfural, 5-methylfurfural and aromatic aldehyde. $R S C A d v$. 2012, 2, 11211-11214.

(21) Wegenhart, B. L.; Yang, L.; Kwan, S. C.; Harris, R.; Kenttämaa H. I.; Abu-Omar, M. M. From furfural to fuel: Synthesis of furoins by organocatalysis and their hydrodeoxygenation by cascade catalysis. ChemSusChem 2014, 7, 2742-2747.

(22) Liu D. J.; Chen, E. Y.-X. Integrated catalytic process for biomass conversion and upgrading to $\mathrm{C}_{12}$ furoin and alkane fuel. ACS Catal. 2014, 4, 1302-1310.

(23) Bremner, J. G. M.; Keeys, R. K. F. The hydrogenation of furfuraldehyde to furfuryl alcohol and sylvan (2-Methylfuran). J. Chem. Soc. 1947, 1068-1080.

(24) Zheng, H.-Y.; Zhu, Y.-L.; Teng, B.-T.; Bai, Z.-Q.; Zhang, C.-H.; Xiang, H.-W.; Li, Y.-W. Towards understanding the reaction pathway in vapour phase hydrogenation of furfural to 2-methylfuran. J. Mol. Catal. A: Chem. 2006, 246, 18-23.

(25) Lange, J.-P.; van der Heide, E.; van Buijtenen, J.; Price, R.; Lange, J.-P.; van der Heide, E.; van Buijtenen, J.; Price, R. Furfural - A Promising Platform for Lignocellulosic Biofuels ChemSusChem 2012, 5, 150-166.

(26) Corma, A.; de la Torre, O.; Renz, M.; Villandier, N. Production of High-Quality Diesel from Biomass Waste Products. Angew. Chem. Int. Ed. 2011, 123, 2375-2378.

(27) Corma, A.; de la Torre, O.; Renz, M. High-quality diesel from hexose- and pentose-derived biomass platform molecules. ChemSusChem 2011, 4, 1574-1577.

(28) Li, G.; Li, N.; Yang, J.; Wang, A.; Wang, X.; Cong Y.; Zhang, T. Synthesis of renewable diesel with the 2-methylfuran, butanal and acetone derived from lignocellulose. Bioresour. Technol. 2013, 134, 66-72.

(29) Li, G.; Li, N.; Li, S.; Wang, A.; Cong, Y.; Wang, X.; Zhang, T.; Synthesis of renewable diesel with hydroxyacetone and 2-methyl-furan. Chem. Commun. 2013, 49, 
$5727-5729$.

(30) Li, G.; Li, N.; Wang, X.; Sheng, X.; Li, S.; Wang, A.; Cong, Y.; Wang, X.; Zhang, T. Synthesis of diesel or jet fuel range cycloalkanes with 2-methylfuran and cyclopentanone from lignocellulose. Energy Fuels 2014, 28, 5112-5118.

(31) Balakrishnan, M.; Sacia, E. R.; Bell, A. T. Syntheses of Biodiesel Precursors: Sulfonic Acid Catalysts for Condensation of Biomass-Derived Platform Molecules. ChemSusChem, 2014, 7, 1078-1085.

(32) Cole, A. C.; Jensen, J. L.; Ntai, I.; Tran, K. L. T.; Weave, K. J.; Forbes, D. C.; Davis, J. H. Novel Brønsted acidic ionic liquids and their use as dual solvent-catalysts. J. Am. Chem. Soc. 2002, 124, 5962-5963.

(33) Li, H.; Zhang, Q.; Liu, X.; Chang, F.; Hu, D.; Zhang, Y.; Xue, W.; Yang, S.; $\mathrm{InCl}_{3}$-ionic liquid catalytic system for efficient and selective conversion of cellulose into 5-hydroxymethylfurfural. $R S C A d v . \mathbf{2 0 1 3}, 3,3648-3654$.

(34) Miao, J.; Wan, H.; Guan, G. Synthesis of immobilized Brønsted acidic ionic liquid on silica gel as heterogeneous catalyst for esterification. Catal. Commun. 2011, $12,353-356$.

(35) Zhen, B.; Jiao, Q.; Zhang, Y.; Wu, Q.; Li, H. Acidic ionic liquid immobilized on magnetic mesoporous silica: preparation and catalytic performance in esterification Appl. Catal. A: Gen. 2012, 445-446, 239-245.

(36) Saravanamurugan, S.; Sujandi, S.; Prasetyanto, E. A.; Park S.-E. Liquid-phase reaction of 2-hydroxyacetophenone and benzaldehyde over $\mathrm{SO}_{3} \mathrm{H}-\mathrm{SBA}-15$ catalysts: influence of microwave and thermal effects. Micropor. Mesopor. Mater. 2008, 112, 97-107.

(37) McDaniel, D. M.; Steinert, L. H.; The $\mathrm{H}_{0}$ values of aqueous selenic acid and their relationship with aqueous vapor pressure. J. Am. Chem. Soc. 1966, 88, 4826-4828.

(38) Yurdakoc, M.; Akcay, M.; Tonbul, Y.; Yurdakoc. K.; Acidity of silica-alumina catalysts by amine titration using Hammett indicators and FT-IR study of pyridine adsorption. Turk. J. Chem. 1999, 23, 319-327.

(39) Saravanamurugan, S.; Van Buu, O. N.; Riisager, A. Conversion of mono- and disaccharides to ethyl levulinate and ethyl pyranoside with sulfonic 
acid-functionalized ionic liquids. ChemSusChem 2011, 4, 723-726.

(40) Saravanamurugan, S.; Riisager, A. Solid acid catalysed formation of ethyl levulinate and ethyl glucopyranoside from mono- and disaccharides. Catal. Commun. 2012, 17, 71-75.

(41) Kiss, A. A.; Dimian A. C.; Rothenberg, G. Solid acid catalysts for biodiesel production- towards sustainable energy. Adv. Synth. Catal., 2006, 348, 75-81.

(42) Li, H.; Govind, K. S.; Kotni, R.; Saravanamurugan, S.; Riisager, A.; Yang, S. Direct catalytic transformation of carbohydrates into 5-ethoxymethylfurfural with acid-base bifunctional hybrid nanospheres Energy Conv. Manage. 2014, 88, $1245-1251$. 


\section{For Table of Contents Use Only}

Catalytic alkylation of 2-methylfuran with formalin using supported acidic ionic liquids

$\mathrm{Hu} \mathrm{Li}$, Shunmugavel Saravanamurugan, Song Yang, and Anders Riisager

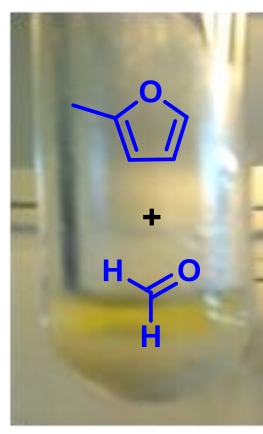

1) SILC

2) Heptane/water

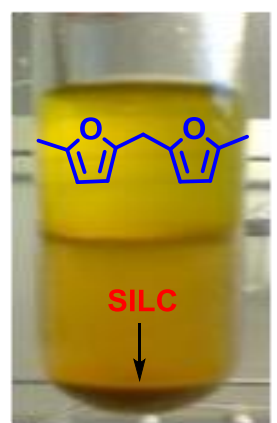

Supported acidic ionic liquid catalysts (SILCs) are efficient and reusable in biphasic alkylation of 2-methylfuran with formalin to form the biofuel precursor bis(5-methylfuran-2-yl)methane (BMFM). 